\title{
optica
}

\section{Enhanced DNA imaging using super-resolution microscopy and simultaneous single-molecule orientation measurements: supplementary material}

\author{
Adam S. Backer, ${ }^{1,2}$ Maurice Y. Lee, ${ }^{1,3}$ W. E. Moerner, ${ }^{1,{ }^{*}}$ \\ ${ }^{1}$ Department of Chemistry, Stanford University, 333 Campus Drive, Stanford CA 94305 \\ ${ }^{2}$ Institute for Computational and Mathematical Engineering, Stanford University, 475 Via Ortega, Stanford CA 94305 \\ ${ }^{3}$ Biophysics Program, Stanford University, Stanford CA 94305 \\ *Corresponding author: wmoerner@stanford.edu
}

Published 17 June 2016

This document provides supplementary information to "Enhanced DNA imaging using super-resolution microscopy and simultaneous single-molecule orientation measurements," http://dx.doi.org/10.1364/optica.3.000659. This supplement consists of additional data in the form of supplemental figures, materials and methods, and calculations. (C) 2016 Optical Society of America

http://dx.doi.org/10.1364/optica.3.000659.s001

\section{Materials and methods}

In this section, we briefly describe our procedures for preparing and imaging samples containing $\lambda$-DNA or single dye molecules embedded in a polymer. $\lambda$-DNA samples were prepared primarily using the 'dynamic molecular combing' technique described in [1]. However, we have also had success using spin-coating to deposit DNA onto coverslips, and by spreading DNA solution between sandwiched coverslips (for example, see [2-4]).

\section{a. Sample preparation}

To prepare $\lambda$-DNA, microscope coverslips were cleaned by blowing them with nitrogen air, and argon etched for ten minutes. Coverslips were immersed in a silanizing solution ( $1 \mathrm{ml}$ methanol, $50 \mu \mathrm{l}$ acetic acid, $10 \mu \mathrm{l}$ 3-aminopropyl-trimethoxysilane) for at least 9 hours, then washed in nanopure water and blown dry with nitrogen air. To stretch DNA onto the coverslips, we used the 'dynamic molecular combing' technique described in [1]. Coverslips were mounted vertically onto a syringe pump (Harvard Apparatus) using a pair of tweezers, and lowered into a DNA solution containing $3 \mu \mathrm{l}$ of stock $\lambda$-DNA solution (500 $\mu \mathrm{g} / \mu \mathrm{l}$, New England Biolabs) in $15 \mathrm{ml}$ of PBS (pH 7.2). After allowing DNA to adhere to the coverslip for 10 min, the syringe pump was used to raise the coverslip out of the DNA solution at a rate of $0.3 \mathrm{~mm} / \mathrm{sec}$. Coverslips were allowed to air-dry for ten minutes, then were loaded into a custom-machined sample holder. For imaging, $200 \mu \mathrm{l}$ of PBS (pH 7.2) and $1 \mu \mathrm{l}$ of $50 \mu \mathrm{M}$ dye solution were pipetted onto the coverslip, and DNA strands were located using low laser power. The dye was subsequently diluted to single-molecule concentrations for PAINT by pipetting out dye/PBS solution in $100 \mu \mathrm{l}$ increments, and pipetting in fresh PBS.

To prepare thin-film samples containing single dye molecules, rhodamine 101 was dissolved first into glycerol to $1 \mathrm{mM}$ concentration, then subsequently diluted to $100 \mathrm{pM}$ into either a toluene and $1 \%$ by wt. poly(methyl methacrylate) (Polysciences) solution, or Mowiol 4-88 poly(vinyl alcohol) (Sigma-Aldrich). Mowiol was prepared from $6 \mathrm{~g}$ of glycerol, $6 \mathrm{ml}$ water, $12 \mathrm{ml}$ of $0.2 \mathrm{M}$ Tris (pH 6.8) and $2.4 \mathrm{~g}$ of Mowiol 4-88. The mixture was warmed to $50^{\circ} \mathrm{C}$ on a hot plate and stirred for 4-5 hours. It was then centrifuged at $5000 \mathrm{Xg}$ for 15 minutes, and $22 \mathrm{ml}$ of supernatant was used for experiments. Ozone-cleaned microscope coverslips were spincoated with dye-polymer solution, and imaged immediately without drying.

\section{b. Imaging using polarization-modulated excitation}

Samples were imaged using either a $561 \mathrm{~nm}$ Sapphire laser (Coherent) for SYTOX Orange/ $\lambda$-DNA and rhodamine 101/polymer samples, or a $641 \mathrm{~nm}$ Cube laser (Coherent) for SiR-Hoechst/ $\lambda$-DNA samples. Once laser light reached the microscope body, it was 
reflected off of a dichroic beamsplitter (ZT405/488/561rpc, Chroma for $561 \mathrm{~nm}$ excitation or FF650-DiO1, Semrock for 641 nm excitation), and focused into the back aperture of the objective lens (Olympus PLAPON60XOSC, 60X/NA1.4 oil). Emitted fluorescence was collected by the same objective, filtered through the dichroic beamsplitter and an emission filter (NF03-561-E, Semrock or HQ675/50m, Chroma), and relayed onto a Si EMCCD (Andor iXon DU-897E). For polarization modulation, we configured an M350-50 electro-optic modulator (Conoptics) as described in [5,6]. To clean up the laser polarization, a linear polarizer was placed before the EOM input (LPVISB050-MP2, Thorlabs). For experiments involving the 641 nm laser, a quarter-wave plate (WPQ05M-633, Thorlabs) was placed after the EOM in order to cause polarization rotation in response to an adjustment of the EOM phase. The $561 \mathrm{~nm}$ laser shared a common excitation pathway with other laser lines, and consequently the laser beam was transmitted through three separate dichroics after the EOM before reaching the microscope body (Di01-R514, Semrock, Q495LP, Chroma, and Di01-R405, Semrock). We found that transmission through these dichroics in combination with reflection off of the dichroic used in the microscope body had a net effect that approximated a quarter-wave plate. Hence, no additional quarter-wave plate was placed after the EOM for experiments involving the $561 \mathrm{~nm}$ laser. (See section 4 for additional details). We used an NI-DAQ PCI 6281 DAC card to supply input voltages to the EOM driver (thus modulating the excitation polarization), and externally triggered our EMCCD in sync with changes in the polarization state. 128 x 128 pixel fields of view were recorded (effective pixel size $121 \mathrm{~nm}$ ) using $40 \mathrm{~ms}$ exposures (54 ms total kinetic cycle time when recording in external trigger mode). DNA samples and rhodamine 101 dye samples were respectively imaged with $5-10 \mathrm{~kW} / \mathrm{cm}^{2}$ and $1-5 \mathrm{~kW} / \mathrm{cm}^{2}$ peak laser intensities.

\section{Measurement precision}

It is well understood that measurements involving fluorescence from single molecules will be corrupted by photon shot-noise. Specifically, we expect localization precision to scale in leading order approximately as $1 / \sqrt{N}$, where $N$ is the number of photons detected over the course of a measurement [7]. In part (a) of this section, we characterize the precisions associated with our $\phi$ and $\gamma$ measurements as a function of detected photons. In part (b), we determine localization precision.

\section{a. Orientation measurement precision}

Dye samples were prepared containing $\sim 100 \mathrm{pM}$ concentrations of rhodamine 101, immobilized in PMMA. Single dye molecules were imaged at low laser power over 50-300 complete polarization cycles (150-900 camera frames). For each polarization cycle, the parameters $\phi$ and $\gamma$ associated with a given molecule were repeatedly measured, and the total number of photons detected over the course of the polarization cycle was computed. The standard deviations in $\phi$ and $\gamma$ measurements ( $\sigma_{\phi}$ and $\sigma_{\gamma}$ respectively) were subsequently computed, and used to estimate measurement precision as a function of detected photons. In order to estimate precision at high photon counts, signals from consecutive camera frames using the same excitation polarization were binned together, and $\left\{\sigma_{\phi}, \sigma_{\gamma}\right\}$ were re-computed. Bins of $2,3,4,5,6,10,12$ and 15 camera frames were used. We note that this procedure forces estimation to be performed at varying levels of background, but this does not significantly influence our measurement because the emitters are much brighter than the background. Precision estimates based on orientation measurements of fourteen separate dye molecules are plotted in Fig. S1. Using least-squares fitting, raw data was fit to the curve:

$$
\sigma_{\phi, \gamma}=\frac{a_{\phi, \gamma}}{\sqrt{N}}
$$

Where $a_{\phi, \gamma}$ is a fitting parameter: $\left\{a_{\phi}=233.9, a_{\gamma}=4.21\right\}$. We estimate precisions of $\left\{\sigma_{\phi}=5.8^{\circ}, \sigma_{\gamma}=0.11\right\}$ for DNA imaging experiments involving the dye SYTOX Orange. For SiR-Hoechst, measurement precisions were $\left\{\sigma_{\phi}=2.5^{\circ}, \sigma_{\gamma}=0.05\right\}$.

From these precision estimates, it is important to appreciate that our capacity to precisely measure the azimuthal orientation of a molecule $\phi$ is significantly superior to our ability to measure the arc-angle $\delta$. For example, using Eq. 2 from the main text, a $\gamma$ measurement of $\gamma=0.8 \pm 0.05$ would imply $\delta=56^{\circ}-73^{\circ}$. Nevertheless, as our experiments involving SiR-Hoechst demonstrate, dramatic differences in $\gamma$ are readily detectable.

\section{b. Localization precision}

Localization precision was estimated directly from our DNA image data assuming effective one-dimensionality of the stretched DNA. Segments of roughly linear DNA approximately $1.5 \mu \mathrm{m}$ in length were selected from our superresolution image data, and the shortest distance $\Delta \mathrm{r}$ between the DNA axis (estimated using spline interpolation as described in section 3 below), and each localization falling near the segment was determined. Localization precision (Fig. S2) was estimated by computing the median absolute deviation in $\Delta \mathrm{r}$ (The sign of $\Delta r$ was assigned based on whether the line connecting a point on the DNA axis to a given localization had positive or negative slope). In order to avoid overweighting spurious dye molecules not attached to the DNA segment of interest, we rejected from our precision calculations all localizations falling more than $50 \mathrm{~nm}$ from the DNA axis, and we use median absolute deviation instead of standard deviation as our figure of merit. The median absolute deviation for DNA labeled with SYTOX Orange was 19.3 $\mathrm{nm}$, and 15.8 for SiR-Hoechst (we measured standard deviations in $\Delta \mathrm{r}$ of $25 \mathrm{~nm}$ and $21 \mathrm{~nm}$ for SYTOX Orange and SiR-Hoechst respectively). As these metrics do not account for localization errors in the direction parallel to the DNA axis, we estimate the true 2D localization precision by multiplying by a factor of $\sqrt{2}$. For SYTOX Orange, we obtain $\sqrt{2} \times 19.3=27.3 \mathrm{~nm}$ and for SiR-Hoechst we

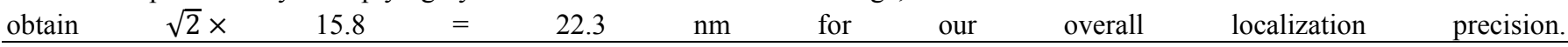


(a)

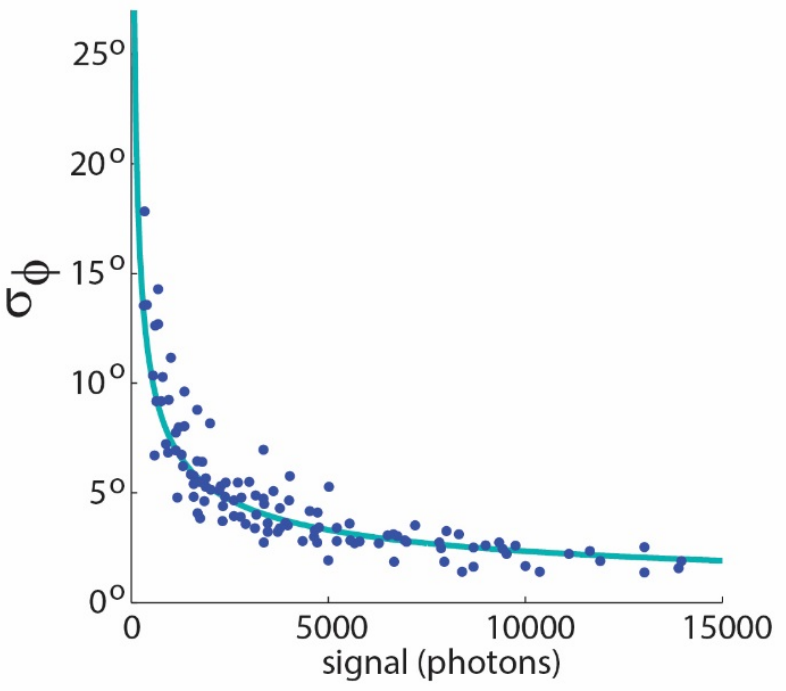

(b)

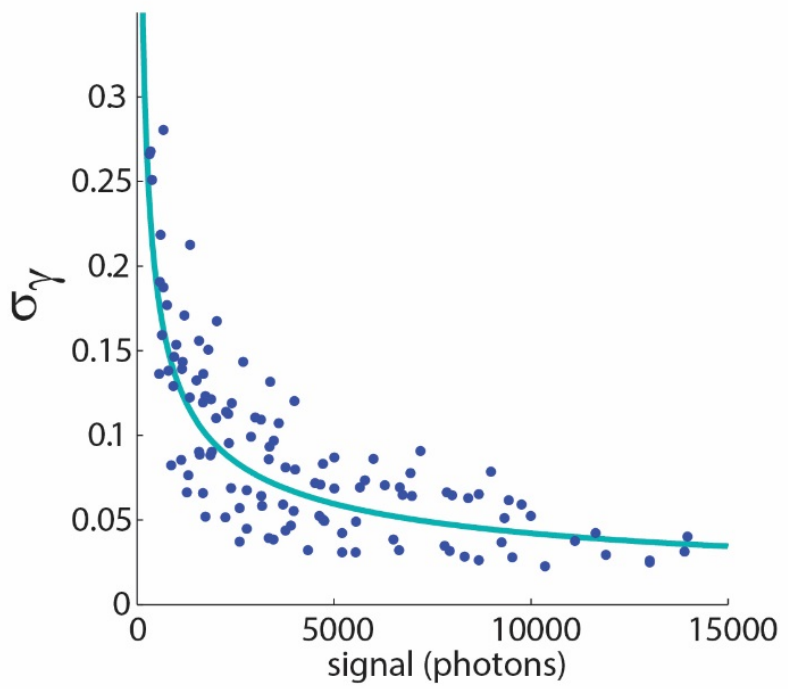

Fig. S1. Orientation and rotational mobility precision measurements. (a) Measurement precision for azimuthal orientation $\phi$ was determined by repeatedly measuring the orientations of 14 different rhodamine 101 molecules in PMMA over 50-300 polarization cycles, and computing standard deviations in the set of measurement results for each molecule. To produce precision estimates corresponding to higher signal levels, successive camera frames acquired using the same excitation polarization were binned together to different degrees as described in section 2. Background varied linearly from approximately 50 photons per frame per pixel (corresponding to 1000 photons of signal) to approximately 700 photons per frame per pixel (10,000 photons of signal). (b) Measurement precision for the rotational immobility parameter $\gamma$ was determined using the same approach as in a.

(a)

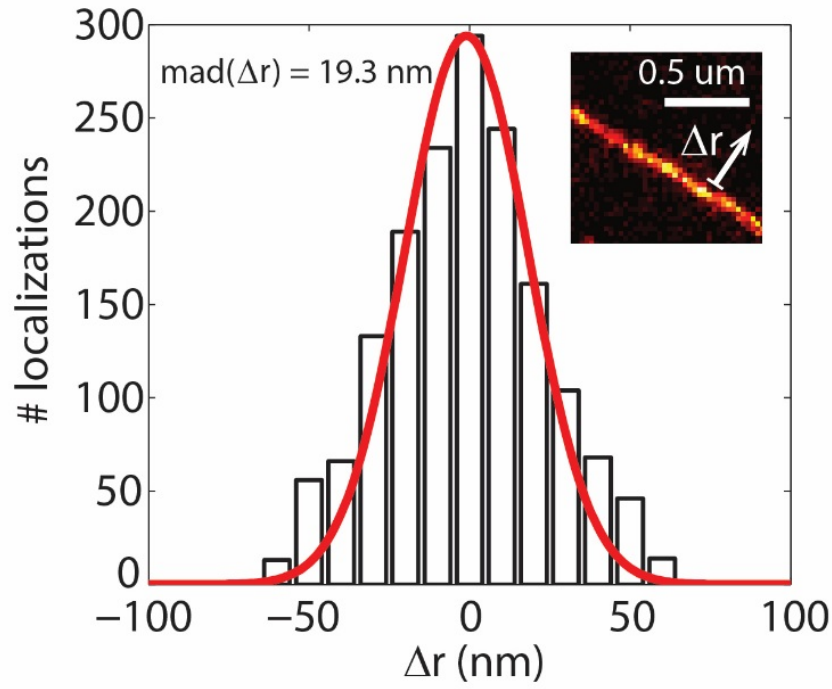

(b)

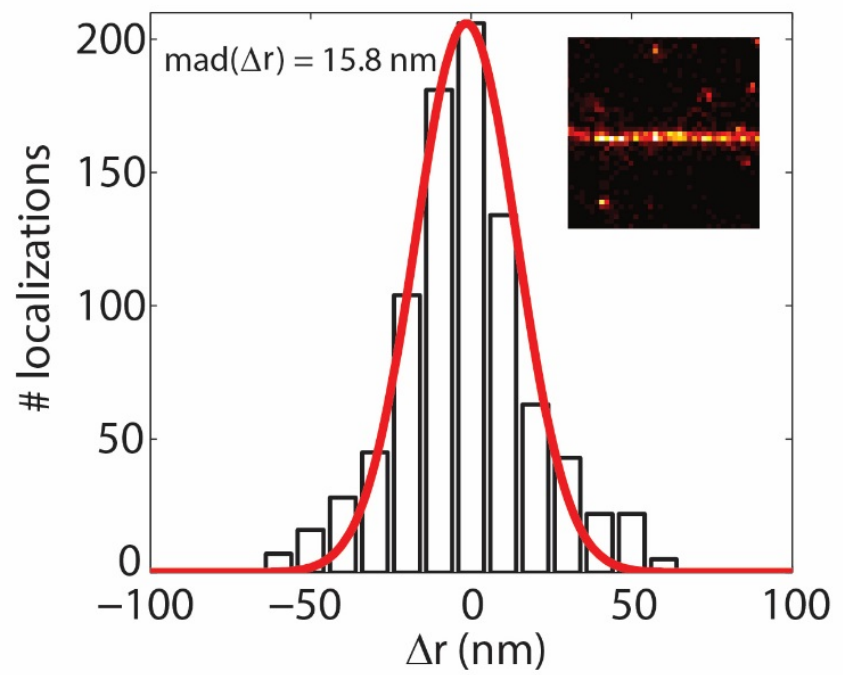

Fig. S2. We estimate localization precision from the median absolute deviation in distances $\Delta \mathrm{r}$ of localizations from the DNA axis, over a $\sim 1.5 \mu \mathrm{m}$ stretch of DNA. (a) For the dye SYTOX Orange, we measure a median absolute deviation of $19.3 \mathrm{~nm}$. (b) For the dye SiR-Hoechst, we measure a median absolute deviation of $15.8 \mathrm{~nm}$.

\section{Overview of image processing pipeline}

In this section, we briefly describe the image processing steps required to obtain single-molecule orientation measurements from raw data. As a pre-processing step, image files were dark-count subtracted and converted from ADC counts to photon counts, using standard EMCCD calibration procedures [8]. In order to subtract fluorescence background arising from diffusing dye molecules in solution, we elected to use temporal median filtering [9]. Since the laser intensity incident upon the sample varied as a function of laser polarization, only camera frames corresponding to the same laser polarization were used for median filtering a given frame of data. We used a filter window of fifty frames before and after the frame of interest. Background-subtracted data was then analyzed using the imageJ plugin quickPALM [10]. The quickPALM software was used to detect and localize single molecules, outputting x/y coordinates and the frame numbers at which molecules appear in the raw data (along with a host of other parameters which were not used for our analysis). From the list of localizations outputted by quickPALM, we subsequently identified molecules that remain 
fluorescent for multiple camera frames by grouping localizations that appear within a distance of less than half a camera pixel (60 nm) over consecutive camera exposure periods. For each molecule, we estimated the total intensity of the fluorescence emission over a given camera frame by drawing a 5-by-5 pixel region of interest (ROI) around the (rounded) coordinates of the localization, and summing the photon counts associated with the thirteen brightest background-subtracted pixels in the ROI. Intensity measurements for a given molecule were sorted according to the excitation polarization. If a molecule remained fluorescent for multiple polarization cycles, the average intensity with respect to each polarization was used for orientation measurements. Additionally, for each molecule, the first and last frame of data for which it was successfully localized were not used for orientation measurements, since it is assumed that the molecule became fluorescent or bleached midway through each of these frames. Hence, only molecules that were successfully localized over a minimum of five consecutive camera frames were incorporated into our orientation datasets. These image processing steps are summarized in Table S1, and orientation measurement results are summarized in Table S2. Localization data was driftcorrected using a redundant cross-correlation algorithm [11], and rendered in $30 \mathrm{~nm}$ voxels color coded in the final figures according to either mean orientation or number of localizations per voxel.

Estimating the axis of DNA filaments required fitting splines to localization data. First, a given DNA filament was selected from our dataset, a ROI was drawn by hand, and a linear fit was performed, incorporating all localizations contained in the ROI. Next, localizations a distance greater than 1 camera pixel $(121 \mathrm{~nm})$ from a point on the fitted line were discarded. A spline curve was then fit to the remaining localizations using the MATLAB built-in function $\mathrm{fit}\left(\mathrm{x}, \mathrm{y},{ }^{\prime}\right.$ smoothingspline'). In order to determine the azimuthal orientations of molecules with respect to the DNA axis, the shortest distance between a given molecule and a point on the spline curve was first determined, and the tangent line to the point on the spline was calculated using finite differences. The azimuthal orientation with respect to the DNA axis was then estimated as the angle between the molecule's orientation measured with respect to the global camera coordinates, and the tangent line of the point along the DNA filament. For the histograms shown in Figs. 2 and 4 (main text), only molecules localized to within $25 \mathrm{~nm}$ of the DNA axis respectively were included. Image processing was performed using customized MATLAB scripts, except for single-molecule detection and localization, for which we used the software quickPALM, as detailed above.

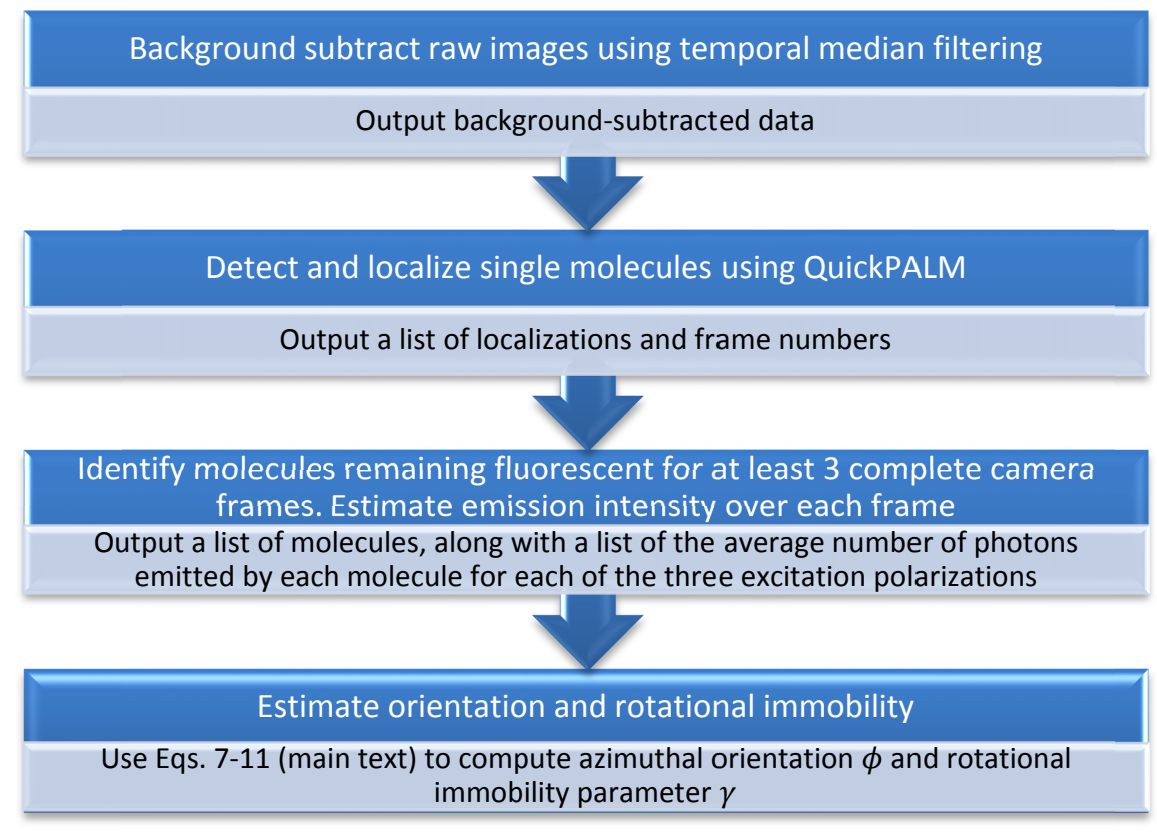

Table S1. The major steps of our orientation estimation procedure are outlined above.

\begin{tabular}{lccc}
\hline \multicolumn{1}{c}{ Dye } & $\begin{array}{c}\tilde{\phi} \\
\text { (w. r. t. DNA axis) }\end{array}$ & $\tilde{\gamma}$ & $\begin{array}{c}\delta \\
\text { (calculated using } \\
\text { Eq. 2, main text) }\end{array}$ \\
\hline \hline SYTOX Orange & $87^{\circ} \pm 18^{\circ}$ & $0.91 \pm 0.16$ & $44^{\circ}$ \\
\hline SiR-Hoechst & $95^{\circ} \pm 42^{\circ}$ & $0.43 \pm 0.27$ & $117^{\circ}$ \\
\hline
\end{tabular}

Table S2. Key results from our DNA imaging experiments are listed above. Median values and median absolute deviations are reported. Azimuthal arc-angle measurements are computed from the immobility parameter $\gamma$ using a rotational diffusion model to determine $\delta$ (See section 5 for derivation). 


\section{Calibration of the excitation polarization}

Our orientation measurement technique requires accurate knowledge of the excitation polarization sequence $\left\{\boldsymbol{E}_{1}, \boldsymbol{E}_{2}, \boldsymbol{E}_{3}\right\}$ which is used to construct the probe matrix $\boldsymbol{P}$. While the probe matrix can handle complex polarization states, for optimal measurement precision we ideally wish to generate three linear pumping polarizations. Unfortunately, the filters and dichroics used in the excitation pathway of our microscope will alter the laser polarization output by the EOM. Specifically, when light that is not entirely p- or spolarized is incident upon a dichroic, its polarization changes due to the fact that the Fresnel reflection coefficients of the dichroic differ significantly for a $45^{\circ}$ angle of incidence. Additionally, dichroics may be constructed from partially birefringent coatings or substrates that further distort the polarization of the laser beam. Therefore, at the sample plane, each excitation polarization can exhibit non-negligible ellipticity. To correct for this effect, and to assemble an accurate probe matrix $\boldsymbol{P}$, it is essential to measure the laser polarization at the microscope objective. We used the following procedure to directly measure the polarization contrast for each of the three desired polarizations:

1. A power-meter detector head is aligned above the microscope objective. A linear polarizer is inserted between the objective and the detector head. The polarizer is rotated such that the angle of maximum transmission, $\eta_{j}$, is determined for each of the $j$ excitation polarizations. This angle is subsequently related to camera-plane coordinates by aligning a Ronchi grating to the polarizer axis, and imaging the grating lines under white light.

2. By rotating the polarizer $90^{\circ}$ from the axis of maximum transmission, each of the $j$ extinction ratios $\varepsilon_{j}$ are measured.

3. To account for the fact that the actual intensity delivered to the sample will vary as a function of polarization, a region of the sample containing only excess dye solution (no linear DNA) is located. The excitation polarization is modulated, and the relative amounts of background intensity $W_{j}$, appearing on the image sensor are measured.

The probe matrix is subsequently assembled as:

$$
\boldsymbol{P}=\boldsymbol{P}_{\|}+\boldsymbol{P}_{\perp}
$$

Where:

$$
\begin{aligned}
& {\left[\begin{array}{lll}
\frac{W_{1} \varepsilon_{1}}{\varepsilon_{1}+1} \cos ^{2}\left(\eta_{1}\right) & \frac{W_{1} \varepsilon_{1}}{\varepsilon_{1}+1} \sin ^{2}\left(\eta_{1}\right) & 2 \frac{W_{1} \varepsilon_{1}}{\varepsilon_{1}+1} \cos \left(\eta_{1}\right) \sin \left(\eta_{1}\right) \\
\frac{W_{2} \varepsilon_{2}}{\varepsilon_{2}+1} \cos ^{2}\left(\eta_{2}\right) & \frac{W_{2} \varepsilon_{2}}{\varepsilon_{2}+1} \sin ^{2}\left(\eta_{2}\right) & 2 \frac{W_{2} \varepsilon_{2}}{\varepsilon_{2}+1} \cos \left(\eta_{2}\right) \sin \left(\eta_{2}\right) \\
\frac{W_{3} \varepsilon_{3}}{\varepsilon_{3}+1} \cos ^{2}\left(\eta_{3}\right) & \frac{W_{3} \varepsilon_{3}}{\varepsilon_{3}+1} \sin ^{2}\left(\eta_{3}\right) & 2 \frac{W_{3} \varepsilon_{3}}{\varepsilon_{3}+1} \cos \left(\eta_{3}\right) \sin \left(\eta_{3}\right)
\end{array}\right]} \\
& \boldsymbol{P}_{\perp}=\left[\begin{array}{ccc}
\frac{W_{1}}{\varepsilon_{1}+1} \cos ^{2}\left(\eta_{1}+90^{\circ}\right) & \frac{W_{1}}{\varepsilon_{1}+1} \sin ^{2}\left(\eta_{1}+90^{\circ}\right) & 2 \frac{W_{1}}{\varepsilon_{1}+1} \cos \left(\eta_{1}+90^{\circ}\right) \sin \left(\eta_{1}+90^{\circ}\right) \\
\frac{W_{2}}{\varepsilon_{2}+1} \cos ^{2}\left(\eta_{2}+90^{\circ}\right) & \frac{W_{2}}{\varepsilon_{2}+1} \sin ^{2}\left(\eta_{2}+90^{\circ}\right) & 2 \frac{W_{2}}{\varepsilon_{2}+1} \cos \left(\eta_{2}+90^{\circ}\right) \sin \left(\eta_{2}+90^{\circ}\right) \\
\frac{W_{3}}{\varepsilon_{3}+1} \cos ^{2}\left(\eta_{3}+90^{\circ}\right) & \frac{W_{3}}{\varepsilon_{3}+1} \sin ^{2}\left(\eta_{3}+90^{\circ}\right) & 2 \frac{W_{3}}{\varepsilon_{3}+1} \cos \left(\eta_{3}+90^{\circ}\right) \sin \left(\eta_{3}+90^{\circ}\right)
\end{array}\right]
\end{aligned}
$$

Our calibration measurements are provided in Table S3.

Our calibration procedure accounts for polarization distortions introduced by the dichroics, removing the possibility of systematic measurement errors. Nevertheless, it is preferable to use a sequence of excitation polarizations that are each as linear as possible. Generation of linear polarization states was achieved using the method described in [5-6]: First, A vertically oriented linear polarizer was placed before the EOM. This polarizer provided extinction ratios in excess of 30,000:1, measured at the EOM input, for both lasers. The EOM was then aligned such that the phase modulation axis of the crystal was oriented at $45^{\circ}$ relative to the input polarizer. Under ideal circumstances, a quarter-wave plate should then be placed after the EOM with fast axis oriented horizontally. The Jones matrix for this idealized system is: 


$$
\begin{aligned}
\boldsymbol{J} & =\frac{1}{2}\left[\begin{array}{ll}
1 & 0 \\
0 & i
\end{array}\right]\left[\begin{array}{cc}
1 & -1 \\
1 & 1
\end{array}\right]\left[\begin{array}{cc}
1 & 0 \\
0 & e^{i \psi}
\end{array}\right]\left[\begin{array}{cc}
1 & 1 \\
-1 & 1
\end{array}\right] \\
& =e^{i \psi / 2}\left[\begin{array}{cc}
\cos (\psi / 2) & -i \sin (\psi / 2) \\
\sin (\psi / 2) & i \cos (\psi / 2)
\end{array}\right]
\end{aligned}
$$

In this configuration, vertically polarized input light will be rotated by an angle $\psi / 2$, where $\psi$ is the variable phase lag introduced by the EOM and output light will be perfectly linearly polarized. For experiments involving the $641 \mathrm{~nm}$ laser, we obtained polarization extinction ratios in excess of 12:1 by using a $633 \mathrm{~nm}$ quarter-wave plate placed after the EOM. However, our $561 \mathrm{~nm}$ laser shared a common beampath with other excitation sources, and was transmitted through a series of dichroics before reaching the sample (see Section 1.b for a complete listing). Due to these additional dichroics, linear polarizations with high extinction ratios (in excess of 50:1) were most readily achieved at the sample without an additional quarterwave plate placed after the EOM. In future experiments, it will be desirable to further improve the linearity of the excitation polarization sequence. Precise polarization compensation may potentially be accomplished using a variable waveplate such as a Babinet-Soleil compensator.

Polarization calibration: $561 \mathrm{~nm}$ Laser Line

\begin{tabular}{cccc}
\hline & $\boldsymbol{E}_{1}$ & $\boldsymbol{E}_{2}$ & $\boldsymbol{E}_{3}$ \\
\hline \hline$\eta$ & $48^{\circ}$ & $98^{\circ}$ & $158^{\circ}$ \\
\hline$W$ & 1.03 & 0.98 & 1 \\
\hline$\varepsilon$ & $300: 1$ & $50: 1$ & $50: 1$ \\
\hline
\end{tabular}

Polarization calibration: $561 \mathrm{~nm}$ Laser Line

\begin{tabular}{cccc}
\hline & $\boldsymbol{E}_{1}$ & $\boldsymbol{E}_{2}$ & $\boldsymbol{E}_{3}$ \\
\hline \hline$\eta$ & $20^{\circ}$ & $80^{\circ}$ & $140^{\circ}$ \\
\hline$W$ & 1 & 1 & 0.96 \\
\hline$\varepsilon$ & $400: 1$ & $315: 1$ & $450: 1$ \\
\hline
\end{tabular}

Polarization calibration: $641 \mathrm{~nm}$ Laser Line

\begin{tabular}{cccc}
\hline & $\boldsymbol{E}_{1}$ & $\boldsymbol{E}_{2}$ & $\boldsymbol{E}_{3}$ \\
\hline \hline$\eta$ & $151^{\circ}$ & $212^{\circ}$ & $91^{\circ}$ \\
\hline$W$ & 1.07 & 0.86 & 1 \\
\hline$\varepsilon$ & $12: 1$ & $12: 1$ & $19: 1$ \\
\hline
\end{tabular}

\section{( $\lambda$-DNA/SYTOX Orange samples)}

$$
\boldsymbol{P}=\left[\begin{array}{ccc}
0.59 & 0.72 & 1.30 \\
0.03 & 1.28 & -0.36 \\
1.08 & 0.18 & -0.87
\end{array}\right] \quad \boldsymbol{P}^{-1}=\left[\begin{array}{ccc}
0.39 & -0.32 & 0.72 \\
0.14 & 0.72 & -0.09 \\
0.52 & -0.25 & -0.27
\end{array}\right]
$$

(Rhodamine 101 dye samples)

$$
\boldsymbol{P}=\left[\begin{array}{ccc}
0.97 & 0.13 & 0.71 \\
0.04 & 1.07 & 0.37 \\
0.62 & 0.44 & -1.04
\end{array}\right] \quad \boldsymbol{P}^{-1}=\left[\begin{array}{ccc}
0.77 & -2.69 & 0.42 \\
-0.16 & 0.87 & 0.20 \\
0.39 & 0.21 & -0.62
\end{array}\right]
$$

\section{( $\lambda$-DNA/SiR-Hoechst samples)}

$$
\boldsymbol{P}=\left[\begin{array}{ccc}
0.76 & 0.29 & -0.76 \\
0.58 & 0.27 & 0.65 \\
0.05 & 0.94 & -0.03
\end{array}\right] \quad \boldsymbol{P}^{-1}=\left[\begin{array}{ccc}
0.72 & 0.82 & -0.46 \\
-0.06 & -0.02 & 1.09 \\
-0.62 & 0.82 & -0.04
\end{array}\right]
$$

Table S3. Calibrations for lasers used in imaging experiments. Amplifier electronics for the EOM were modified between $\lambda$ DNA/SYTOX Orange experiments and rhodamine 101 measurements, hence a new calibration was performed and a different $\boldsymbol{P}$ matrix was $\quad$ used $\quad$ for $\quad$ data analysis.

\section{Interpretation of $\phi$ and $\gamma$ using an azimuthal rotational diffusion constraint}

In this section, we apply a rotational diffusion model similar to that used in [12] to relate our measured parameter $\gamma$ to an azimuthal 'arc-angle' $\delta$, which describes the range of azimuthal orientations that a molecule visits. According to this model (Fig. S3(a)), we assume that over the course of a measurement period, the polar orientation of each molecule remains constant-in other words, each molecule remains fixed at its mean polar orientation $\theta_{0}$, but the parameter $\theta_{0}$ may differ from molecule to molecule. In addition, we mandate that the rotational diffusion of each molecule is an ergodic process. More general rotational diffusion models assuming both variable polar and azimuthal orientation have been considered in [13-15], and will be analyzed in section 6. However, since our imaging technique does not directly measure $\theta_{0}$, in this section we will accordingly restrict our analysis to a simplified model in which polar orientation is assumed to be constant. In future experiments, z-polarized excitation light may be introduced using totalinternal reflection (as is done in e. g. [16-19]) or annular illumination, permitting accurate measurement of $\theta_{0}$ in addition to the 3D cone angle, albeit at the expense of a longer sequence of excitation polarizations. As previously considered by [12], we use the following steady-state probability distribution $P_{s s}\left(\phi^{\prime} \mid \phi_{0}, \delta\right)$ to describe a molecule's rotational mobility:

$$
P_{s s}\left(\phi^{\prime} \mid \phi_{0}, \delta\right)=\left\{\begin{array}{cc}
\frac{1}{\delta} & \text { if } \phi_{0}-\frac{\delta}{2}<\phi^{\prime}<\phi_{0}+\frac{\delta}{2} \\
0 & \text { Otherwise }
\end{array}\right.
$$


That is, a given molecule is constrained to rotate about its mean azimuthal orientation $\phi_{0}$ up to an arc half-aperture angle of $\delta / 2$. Using the assumption of ergodicity, the temporal integral (Eq. 1, main text) relating measured intensity to an excitation polarization $\boldsymbol{E}$ may be re-written as a spatial integral:

$$
U=\frac{A}{T} \int_{0}^{T}\left|\boldsymbol{\mu}^{\dagger}(t) \boldsymbol{E}\right|^{2} d t=\frac{A}{\delta} \int_{\phi_{0}-\delta / 2}^{\phi_{0}+\delta / 2}\left|\boldsymbol{\mu}^{\dagger}\left(\phi^{\prime}, \theta_{0}\right) \boldsymbol{E}\right|^{2} d \phi^{\prime}
$$

Using the assumption that the polar orientation is constant, in addition to the fact that the z-polarization component of the electric field $\boldsymbol{E}$ is negligible permits Eq. (S5.2) to simplified:

$$
U=\frac{A}{\delta} \sin ^{2}\left(\theta_{0}\right) \boldsymbol{E}_{x y}^{\dagger}\left(\int_{\phi_{0}-\delta / 2}^{\phi_{0}+\delta / 2}\left[\begin{array}{cc}
\cos ^{2}\left(\phi^{\prime}\right) & \cos \left(\phi^{\prime}\right) \sin \left(\phi^{\prime}\right) \\
\cos \left(\phi^{\prime}\right) \sin \left(\phi^{\prime}\right) & \sin ^{2}\left(\phi^{\prime}\right)
\end{array}\right] d \phi^{\prime}\right) \boldsymbol{E}_{x y}
$$

Where, as in the main text, $\boldsymbol{E}_{x y}$ denotes a vector containing the x and y components of the excitation field $\boldsymbol{E}$. Evaluating the integral in Eq. (S5.3) using the rotated coordinate system $\phi^{R}=\phi^{\prime}-\phi_{0}$ yields:

$$
\begin{aligned}
U & =\frac{A}{\delta} \sin ^{2}\left(\theta_{0}\right) \boldsymbol{E}^{\dagger} \boldsymbol{R}_{\phi_{0}}\left(\int_{-\delta / 2}^{\delta / 2}\left[\begin{array}{cc}
\cos ^{2}\left(\phi^{R}\right) & 0 \\
0 & \sin ^{2}\left(\phi^{R}\right)
\end{array}\right] d \phi^{R}\right) \boldsymbol{R}_{\phi_{0}}^{\dagger} \boldsymbol{E} \\
& =\frac{A}{\delta} \sin ^{2}\left(\theta_{0}\right) \boldsymbol{E}^{\dagger} \boldsymbol{R}_{\phi_{0}}\left[\begin{array}{cc}
\frac{\delta+\sin (\delta)}{2} & 0 \\
0 & \frac{\delta-\sin (\delta)}{2}
\end{array}\right] \boldsymbol{R}_{\phi_{0}}^{\dagger} \boldsymbol{E}
\end{aligned}
$$

Where the rotation matrix $\boldsymbol{R}_{\phi_{0}}$ is:

$$
\boldsymbol{R}_{\phi_{0}}=\left[\begin{array}{cc}
\cos \left(\phi_{0}\right) & -\sin \left(\phi_{0}\right) \\
\sin \left(\phi_{0}\right) & \cos \left(\phi_{0}\right)
\end{array}\right]
$$

Hence, using three separate intensity measurements taken with three separate excitation polarizations, the measurement matrix $\boldsymbol{M}_{x y}$ will be acquired:

$$
\boldsymbol{M}_{x y}=\frac{A}{\delta} \sin ^{2}\left(\theta_{0}\right) \boldsymbol{R}_{\phi_{0}}\left[\begin{array}{cc}
\frac{\delta+\sin (\delta)}{2} & 0 \\
0 & \frac{\delta-\sin (\delta)}{2}
\end{array}\right] \boldsymbol{R}_{\phi_{0}}^{\dagger}
$$

Since the rotation matrices $\boldsymbol{R}_{\phi_{0}}$ do not alter the eigenvalues of $\boldsymbol{M}_{x y}$, we observe that the eigenvalues $\left\{\lambda_{1}, \lambda_{2}\right\}$ are:

$$
\begin{aligned}
& \lambda_{1}=\frac{A}{\delta} \sin ^{2}\left(\theta_{0}\right)\left(\frac{\delta+\sin (\delta)}{2}\right) \\
& \lambda_{2}=\frac{A}{\delta} \sin ^{2}\left(\theta_{0}\right)\left(\frac{\delta-\sin (\delta)}{2}\right)
\end{aligned}
$$

Furthermore, the eigenvectors $\left\{\boldsymbol{v}_{1}, \boldsymbol{v}_{2}\right\}$ of $\boldsymbol{M}_{x y}$ are given by the columns of the rotation matrix $\boldsymbol{R}_{\phi_{0}}$. From Eq. (S5.7), the rotational immobility $\gamma$ is computed:

$$
\gamma=\frac{\lambda_{1}-\lambda_{2}}{\lambda_{1}+\lambda_{2}}=\frac{\sin (\delta)}{\delta}
$$


Thus recovering our formula (main text Eq. 2) for the azimuthal arc-angle $\delta$. Now, consider a vector pointing in the direction of the mean azimuthal orientation: $\hat{\boldsymbol{v}}=\left[\begin{array}{c}\cos \left(\phi_{0}\right) \\ \sin \left(\phi_{0}\right)\end{array}\right]$. We verify:

$$
\boldsymbol{M}_{x y} \hat{\boldsymbol{v}}=\frac{A}{\delta} \sin ^{2}\left(\theta_{0}\right)\left(\frac{\delta+\sin (\delta)}{2}\right) \hat{\boldsymbol{v}}=\lambda_{1} \boldsymbol{v}_{1}
$$

Hence, we have confirmed that the eigenvector corresponding to the maximum eigenvalue of $\boldsymbol{M}_{x y}$ points in the direction of the mean azimuthal orientation $\phi_{0}$. Therefore, the parameter $\phi$, which is measured over the course of our experiments, is equivalent to the mean orientation $\phi_{0}$.
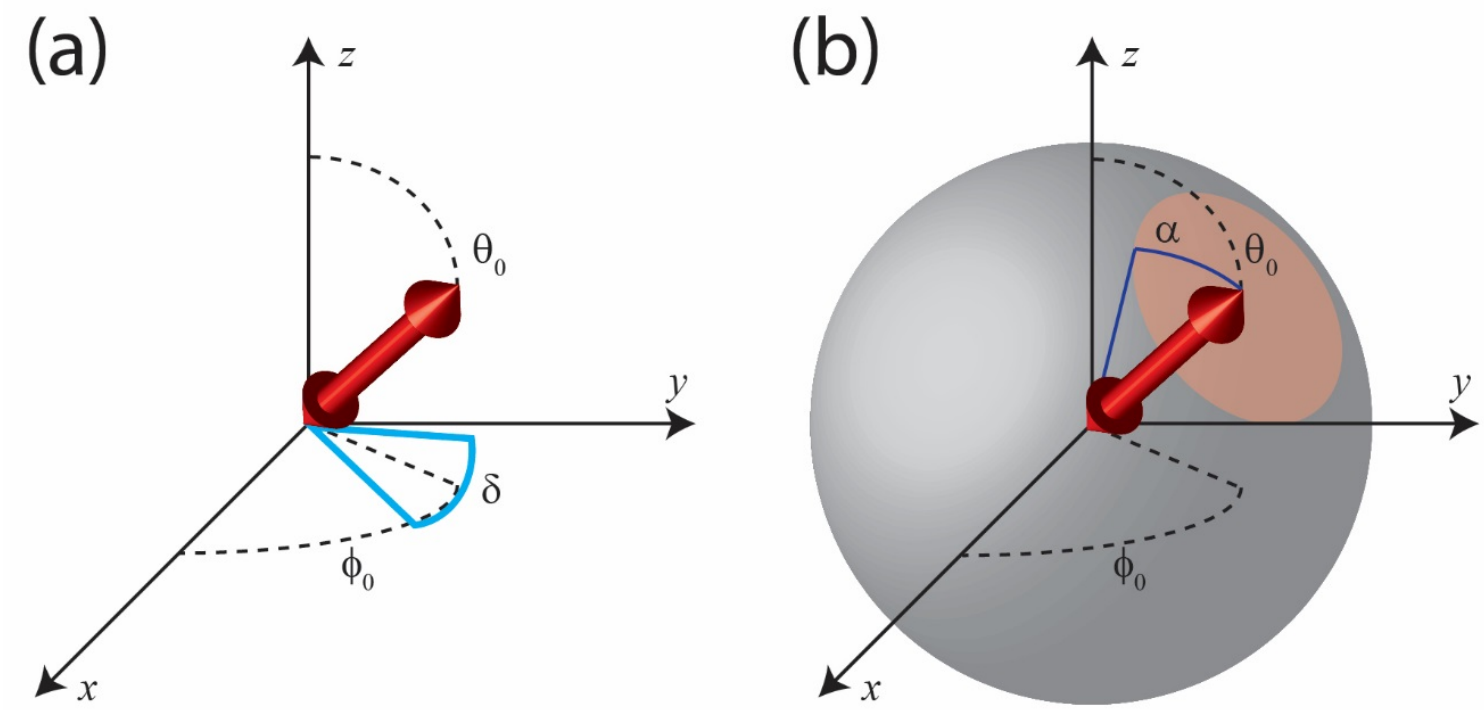

Fig. S3. Theoretical models for rotational diffusion calculations. (a) According to the rotational diffusion model used throughout the main text of this paper and discussed in section 5, the absorption dipole moment is assumed to remain at a constant polar orientation $\theta_{0}$ throughout the measurement sequence, but may wobble about the mean azimuthal orientation $\phi_{0}$, within the full aperture described by $\delta$. In this case, the azimuthal arc-angle $\delta$ is determined by $\gamma$ alone, and may be measured on a molecule-by-molecule basis. (b) According to the three-dimensional rotational diffusion model discussed in section 6, the absorption dipole is constrained to rotate within a half-aperture defined by the angle $\alpha$, and the polar orientation is no longer assumed to remain constant throughout the measurement period.

\section{Extended analysis of experimentally measured parameters using a three- dimensional rotational diffusion constraint}

In the previous section, we used a rotational diffusion model that assumes constant polar orientation to derive simple expressions relating the measured parameters $\{\phi, \gamma\}$ to mean azimuthal orientation $\phi_{0}$ and an azimuthal arc-angle $\delta$. Use of a constant polar orientation constraint was appealing, as it enabled a straightforward and unambiguous interpretation of the primary experimental results. However, a more physically realistic rotational diffusion model allows for the polar orientation of a molecule to vary as well as its azimuthal orientation over the course of a single measurement period [13-15], and this more complex, 3D constraint may be needed in some cases. In this section, we interpret our experimental measurements $\{\phi, \gamma\}$ in the context of the more realistic $3 \mathrm{D}$ model depicted in Fig. S3(b). Here, we assume that a given molecule has a mean orientation given by the angles $\left\{\phi_{0}, \theta_{0}\right\}$. However, the molecule is free to 'wobble' about its mean orientation within a cone prescribed by the half-aperture cone-angle $\alpha$. Over the course of our analysis, we will show that the measured parameter $\phi$ is in fact equivalent to the mean azimuthal orientation $\phi_{0}$ of a molecule rotating within a $3 \mathrm{D}$ cone. This result is in agreement with our analysis from the previous section. No assumptions or approximations regarding $\theta_{0}$ are required in order to obtain an accurate measurement of $\phi_{0}$. However, in contrast to the model studied in section 5, for the 3D model analyzed here, the immobility parameter $\gamma$ is a function of both the cone-angle $\alpha$ and mean polar orientation $\theta_{0}$. See part (b) of this section for details.

The theoretical analysis contained in this section permits us to quantitatively estimate the extent to which orientation-induced errors degrade our localization accuracy. Over the past years, a number of researchers have determined [20-22] that systematic localization errors are incurred when attempting to estimate the positions of rotationally constrained fluorophores using a simple Gaussian model for the point-spread function (PSF) or centroid-based estimation schemes. However, it was also pointed out that rotationally mobile fluorophores will produce more symmetric PSFs, and will yield more accurate localizations [13]. From our 
measurements of the immobility parameter $\gamma$, we estimate that localization errors are bounded to $35 \mathrm{~nm}$ when using the intercalating dye SYTOX Orange to label DNA. Localization errors are bounded to $16 \mathrm{~nm}$ for the dye SiR-Hoechst, owing to its enhanced rotational freedom. See part (c) of this section for details.

\section{a. Theoretical model}

Formally, following [13-15] we describe the steady-state probability distribution of a given molecule's absorption dipole orientation as:

$$
P_{s s}\left(\phi^{\prime}, \theta^{\prime} \mid \phi_{0}, \theta_{0}, \alpha\right)=\left\{\begin{array}{cc}
\frac{1}{2 \pi(1-\cos (\alpha))} & \text { if } \boldsymbol{\mu}^{\dagger}\left(\phi^{\prime}, \theta^{\prime}\right) \boldsymbol{\mu}\left(\phi_{0}, \theta_{0}\right)>\cos (\alpha) \\
0 & \text { Otherwise }
\end{array}\right.
$$

Assuming ergodicity, we calculate the total intensity $U$ of light emitted by a given molecule as:

$$
\begin{aligned}
U & =A \int_{0}^{2 \pi} \int_{0}^{\pi} P_{s s}\left(\phi^{\prime}, \theta^{\prime} \mid \phi_{0}, \theta_{0}, \alpha\right)\left|\boldsymbol{\mu}^{\dagger}\left(\phi^{\prime}, \theta^{\prime}\right) \boldsymbol{E}\right|^{2} \sin \left(\theta^{\prime}\right) d \theta^{\prime} d \phi^{\prime} \\
& =A \boldsymbol{E}^{\dagger}\left(\int_{0}^{2 \pi} \int_{0}^{\pi} P_{s s}\left(\phi^{\prime}, \theta^{\prime} \mid \phi_{0}, \theta_{0}, \alpha\right) \boldsymbol{\mu}\left(\phi^{\prime}, \theta^{\prime}\right) \boldsymbol{\mu}^{\dagger}\left(\phi^{\prime}, \theta^{\prime}\right) \sin \left(\theta^{\prime}\right) d \theta^{\prime} d \phi^{\prime}\right) \boldsymbol{E} \\
& =A \boldsymbol{E}^{\dagger} \boldsymbol{M}_{s s} \boldsymbol{E}
\end{aligned}
$$

In the third line of Eq. (S6.2), $\boldsymbol{M}_{s s}$ denotes the 3-by-3 matrix that results from evaluating the integral contained in parentheses on the second line (see [15] for details). Unlike the model considered in section 5, our analysis of the 3D rotational diffusion model is complicated by the following feature: Over the course of a single camera exposure, a molecule will presumably emit photons at different polar orientations $\theta^{\prime}$. However, the probability that a photon will be detected will also vary as a function of $\theta^{\prime}$. In order to properly account for the $\theta^{\prime}$-dependent collection efficiency of the objective, it is necessary to calculate the orientation-dependent PSF for a given molecule using vectorial diffraction theory. As previously detailed in [15], the PSF of a partially mobile molecule may be calculated using a judicious superposition of the electric fields associated with rotationally fixed dipoles aligned along the $\mathrm{x}, \mathrm{y}$ and $\mathrm{z}$ coordinate axes. Let us define two 3-dimensional vectors $\boldsymbol{u}_{x}(\boldsymbol{r})$ and $\boldsymbol{u}_{y}(\boldsymbol{r})$. The elements of a given vector contain the electric fields associated with an $\mathrm{x}, \mathrm{y}$, or $\mathrm{z}$ oriented dipole at a given point $\boldsymbol{r}$ in the image plane, while the subscripts $\{x, y\}$ denote the respective $\mathrm{x}$ and $\mathrm{y}$ polarized contributions to the electric field. (At the image plane, the NA of the optical system has been reduced tremendously owing to the long focal length of the microscope tube lens, therefore the z-polarized contribution to the electric field is negligible.) Hence, the overall PSF is computed as:

$$
\begin{aligned}
P S F(\boldsymbol{r}) & =U \sum_{j=x, y} \boldsymbol{u}_{j}^{\dagger}(\boldsymbol{r})\left(\int_{0}^{2 \pi} \int_{0}^{\pi} P_{e m i t}\left(\phi^{\prime}, \theta^{\prime} \mid \phi_{0}, \theta_{0}, \alpha\right) \boldsymbol{\mu}\left(\phi^{\prime}, \theta^{\prime}\right) \boldsymbol{\mu}^{\dagger}\left(\phi^{\prime}, \theta^{\prime}\right) \sin \left(\theta^{\prime}\right) d \theta^{\prime} d \phi^{\prime}\right) \boldsymbol{u}_{j}(\boldsymbol{r}) \\
& =U \sum_{j=x, y} \boldsymbol{u}_{j}^{\dagger}(\boldsymbol{r}) \boldsymbol{M}_{e m i t} \boldsymbol{u}_{j}(\boldsymbol{r})
\end{aligned}
$$

In Eq. (S6.3), $P_{\text {emit }}\left(\phi^{\prime}, \theta^{\prime} \mid \phi_{0}, \theta_{0}, \alpha\right)$ is the probability of a molecule emitting a photon at a given orientation $\left\{\phi^{\prime}, \theta^{\prime}\right\}$, and $\boldsymbol{M}_{\text {emit }}$ is the 3-by-3 matrix that results from evaluating the integral contained in parentheses. The total intensity detected by an image sensor $U_{d e t}$ is calculated by summing the intensities of the individual pixels in the region-of-interest used to record $P S F(\boldsymbol{r})$. In general, the emission probability distribution $P_{\text {emit }}\left(\phi^{\prime}, \theta^{\prime} \mid \phi_{0}, \theta_{0}, \alpha\right)$ depends on the relative durations of a given molecule's fluorescence lifetime $\tau_{f}$, and rotational correlation time $\tau_{r}$. Determining $P_{e m i t}\left(\phi^{\prime}, \theta^{\prime} \mid \phi_{0}, \theta_{0}, \alpha\right)$ from first principles requires solving the rotational diffusion equation using boundary conditions consistent with $P_{s s}\left(\phi^{\prime}, \theta^{\prime} \mid \phi_{0}, \theta_{0}, \alpha\right)$, as was done in [13]. In order to streamline our analysis, we will consider only two limiting cases in this text: That of 'fast' rotational diffusion $\left(\tau_{f}>>\tau_{r}\right)$ and that of 'slow' rotational diffusion $\left(\tau_{f}<<\tau_{r}\right)$. By explicitly calculating only the point-spread functions $P S F_{\text {fast }}(\boldsymbol{r})$ and $P S F_{\text {slow }}(\boldsymbol{r})$ associated with these two limiting regimes, the emission probability distributions become significantly simpler to handle, and lengthy numerical calculations are avoided. Furthermore, a key theoretical result from [14] guarantees that when $\tau_{r}$ is of comparable magnitude to $\tau_{f}$, The resulting PSF may be computed from the formula:

$$
P S F(\boldsymbol{r})=\left(\frac{\tau_{f}}{\tau_{f}+\tau_{r}}\right) P S F_{\text {fast }}(\boldsymbol{r})+\left(\frac{\tau_{r}}{\tau_{f}+\tau_{r}}\right) P S F_{\text {slow }}(\boldsymbol{r})
$$


Hence it is sufficient to calculate the point-spread functions $P S F_{\text {fast }}(\boldsymbol{r})$ and $P S F_{\text {slow }}(\boldsymbol{r})$ associated with a rapidly and slowly rotating molecule respectively, then use the results from these limiting cases to generate the point-spread function for arbitrary $\tau_{r}$ and $\tau_{f}$. While Eq. (S6.4) is appealingly simple, its derivation is by no means trivial. The reader is referred to [14] for the details. Throughout our analysis, we will also assume that the absorption dipole moment of the dyes used in our experiments are parallel to the emission dipole moments, as was done in [13].

In the limit of fast rotational diffusion $\left(\tau_{f} \gg \tau_{r}\right)$ a molecule's orientation upon emission will not be biased by its orientation upon absorption. That is, the emission probability distribution will have sufficient time to 'relax' into the steady-state probability distribution:

$$
P_{\text {emit }}^{\text {fast }}\left(\phi^{\prime}, \theta^{\prime} \mid \phi_{0}, \theta_{0}, \alpha\right)=P_{s s}\left(\phi^{\prime}, \theta^{\prime} \mid \phi_{0}, \theta_{0}, \alpha\right)
$$

On the other hand, in the limit of slow rotational diffusion $\left(\tau_{f}<<\tau_{r}\right)$, a molecule's orientation upon emission is identical to its orientation upon absorption, and the emission probability distribution is identical to the absorption probability distribution:

$$
P_{\text {emit }}^{\text {slow }}\left(\phi^{\prime}, \theta^{\prime} \mid \phi_{0}, \theta_{0}, \alpha\right)=\frac{P_{s s}\left(\phi^{\prime}, \theta^{\prime} \mid \phi_{0}, \theta_{0}, \alpha\right)\left|\boldsymbol{\mu}^{\dagger}\left(\phi^{\prime}, \theta^{\prime}\right) \boldsymbol{E}\right|^{2}}{\int_{0}^{2 \pi} \int_{0}^{\pi} P_{s s}\left(\phi^{\prime \prime}, \theta^{\prime \prime} \mid \phi_{0}, \theta_{0}, \alpha\right)\left|\boldsymbol{\mu}^{\dagger}\left(\phi^{\prime \prime}, \theta^{\prime \prime}\right) \boldsymbol{E}\right|^{2} \sin \left(\theta^{\prime \prime}\right) d \theta^{\prime \prime} d \phi^{\prime \prime}}
$$

By substituting either Eq. (S6.5) or Eq. (S6.6) for the emission probability distribution in Eq. (S6.3), $P S F_{\text {fast }}(\boldsymbol{r})$ and $P S F_{\text {slow }}(\boldsymbol{r})$ are respectively calculated. The integral in the first line of Eq. (S6.3) may be done analytically, but is quite tedious. For our simulations, the integrations required to compute the matrices $\boldsymbol{M}_{s s}$ and $\boldsymbol{M}_{\text {emit }}$ were performed using the MATLAB symbolic math toolbox. The polarized dipole electric fields $\boldsymbol{u}_{x}(\boldsymbol{r})$ and $\boldsymbol{u}_{y}(\boldsymbol{r})$ were calculated for a dipole embedded at a water-glass interface, assuming a 1.4 NA oil objective lens, and an effective camera pixel size of $121 \mathrm{~nm}$ (details for our simulation of dipole electric fields may be found in the appendix of [23]).

\section{b. Interpretation of the measurements $\phi$ and $\gamma$}

In part (a) of this section, we described how the PSF for a given molecule may be calculated in the limits of fast and slow rotational diffusion, for arbitrary excitation polarization $\boldsymbol{E}$. Here, we leverage these calculations to better understand the meaning of the measured parameters $\phi$ and $\gamma$ in the context of a 3D rotational diffusion model. We performed simulations in which molecules were sequentially excited using an electric field polarized at $0^{\circ}, 60^{\circ}$, and $120^{\circ}$ in the $\mathrm{x}-\mathrm{y}$ plane. For each excitation polarization a simulated image $P S F(\boldsymbol{r})$ was generated using Eq. (S6.3), and the total detected intensity $U_{\text {det }}$ was computed. For our first round of simulations, we set the parameters $\theta_{0}=45^{\circ}$ and $\alpha=30^{\circ}$, and generated a corpus of single-molecule intensity measurements by varying the parameter $\phi_{0}$ from $0^{\circ}$ to $180^{\circ}$. For each distinct $\phi_{0}$, the measured parameter $\phi$ was calculated using the procedure described in the main text, based on the total intensity contained in each of the simulated images. Fig. S4(a) plots results for both the limit of fast and slow rotational diffusion. From our simulations, the mean azimuthal orientation $\phi_{0}$ corresponds to the measured parameter $\phi$ exactly. (This result was tested, and remains valid for different values of $\theta_{0}$ and $\alpha$.) Next, we wished to investigate the parameter $\gamma$. A new round of simulations was performed in which we set $\phi_{0}=0^{\circ}$ and varied $\theta_{0}$ from $0^{\circ}$ to $90^{\circ}$. The cone angles $\alpha=\left\{20^{\circ}, 40^{\circ}, 60^{\circ}\right\}$ were used. For each distinct $\theta_{0}$ the parameter $\gamma$ was computed (simulations were repeated using different choices of $\phi_{0}$, and it was verified that results do not change). Fig. S4(b) plots results for both the limit of fast and slow rotational diffusion. These plots demonstrate that $\gamma$ is a function of both $\theta_{0}$ and $\alpha$. Hence, in order to obtain an accurate measurement of the 3D coneangle $\alpha$, it is necessary to obtain an independent estimate of the polar orientation $\theta_{0}$. It is important to note that the immobility parameter $\gamma$ increases monotonically with respect to $\theta_{0}$. This is due to the fact that for a fixed cone-angle $\alpha$, a given molecule explores a reduced range of azimuthal orientations when its mean orientation lies close to the $\mathrm{x}-\mathrm{y}$ plane. This feature underscores the fact that the parameter $\gamma$ is a measure of only the azimuthal wobble experienced by a given molecule.

\section{c. Assessment of orientation-induced localization errors}

The ability to accurately estimate the positions of single fluorescent molecules is critical to localization-based super-resolution microscopy. Unfortunately, rotationally constrained single molecules produce PSFs that are asymmetric, and vary as a function of both orientation and microscope defocus. The overall prevalence of orientation-induced localization errors has been a highly debated subject [13,22], and has inspired a variety of innovative correction schemes [24-26]. In this section, we estimate the magnitude of the errors incurred when using the dyes SYTOX Orange and SiR-Hoechst to image DNA. From our experimentally obtained $\gamma$ 
measurements, we (roughly) approximate the average 3D cone-angle $\alpha$ as $\delta / 2$. Hence for SYTOX Orange, we estimate $\alpha=22^{\circ}$, and for SiR-Hoechst we estimate $\alpha=58.5^{\circ}$. A sequence of PSFs were simulated using these cone-angles, and values of $\theta_{0}$ were varied from $0^{\circ}$ to $90^{\circ}$. A microscope defocus of $100 \mathrm{~nm}$ was assumed, and emission wavelengths of $580 \mathrm{~nm}$ and $670 \mathrm{~nm}$ were used for SYTOX Orange and SiR-Hoechst respectively. PSFs were simulated in the limit of fast and slow rotational diffusion. For the case of slow rotational diffusion, images were simulated using excitation polarized either parallel or perpendicular to $\phi$. PSFs were fitted using Gaussian model functions and resulting localization errors were plotted as a function of $\theta_{0}$. Results are shown in Fig. S5.

According to our calculations, the largest localization errors are incurred for values of $\theta_{0}$ between $20^{\circ}$ and $40^{\circ}$. Errors are expected to be significantly larger for SYTOX Orange (a maximum of $35 \mathrm{~nm}$ as opposed to $16 \mathrm{~nm}$ for SiR-Hoechst), owing to the significantly larger $\gamma$ measurements. However, overall, we note that our calculations yield significantly smaller localization errors than previously predicted in $[13,22]$. This feature is due to the fact that we assume a relatively modest microscope defocus of $100 \mathrm{~nm}$. Since our measurements are performed on a nearly two-dimensional sample consisting of DNA adhered to a microscope coverslip, we expect the majority of detected molecules to reside close to the focal plane of the objective. Any defocus-induced localization error incurred will be primarily a result of stage-drift, not the geometry of the sample itself. Hence for this sample, it is possible to maintain relatively high localization accuracy, even though we are imaging rotationally immobilized fluorophores.

(a)

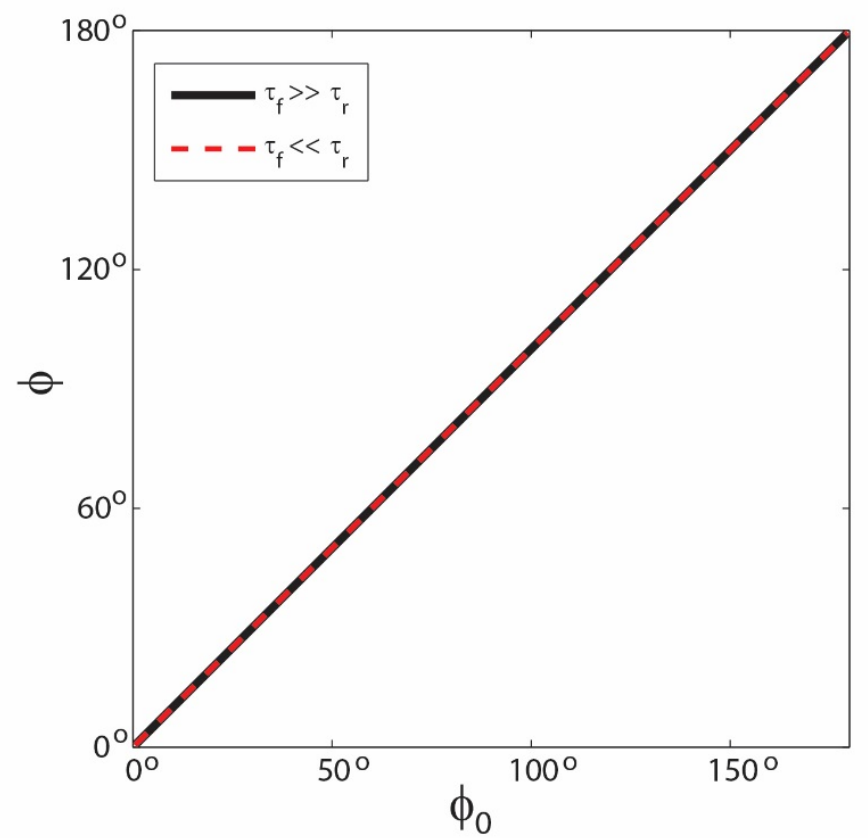

(b)

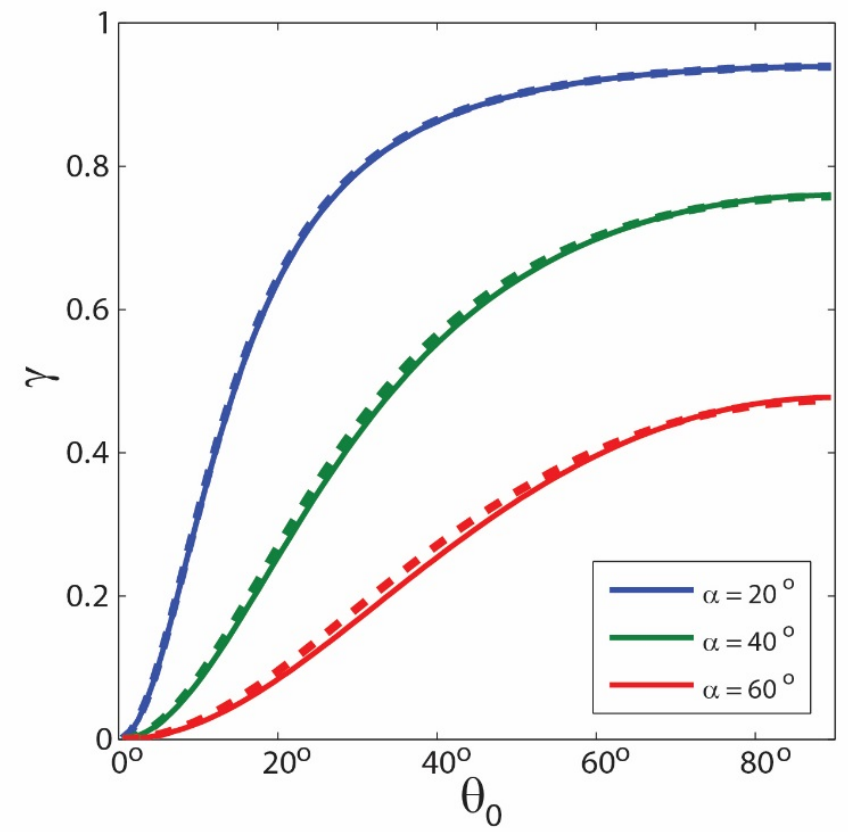

Fig. S4. Theoretical analysis of the 3D rotational diffusion model as described in section 6. (a) The experimentally measured parameter $\phi$ is equivalent to the mean azimuthal orientation $\phi_{0}$, regardless of the unknown mean polar orientation $\theta_{0}$, and the unknown 3D cone angle $\alpha$. This result is valid in the limit of both fast $\left(\tau_{f}>>\tau_{r}\right)$ and slow $\left(\tau_{f}<<\tau_{r}\right)$ rotational diffusion (solid and dotted lines respectively). (b) The immobility parameter $\gamma$ varies both as a function of cone angle $\alpha$ and mean polar orientation $\theta_{0}$. Calculations performed in the limit of fast and slow rotational diffusion are plotted using solid and dotted lines respectively. 
(a)

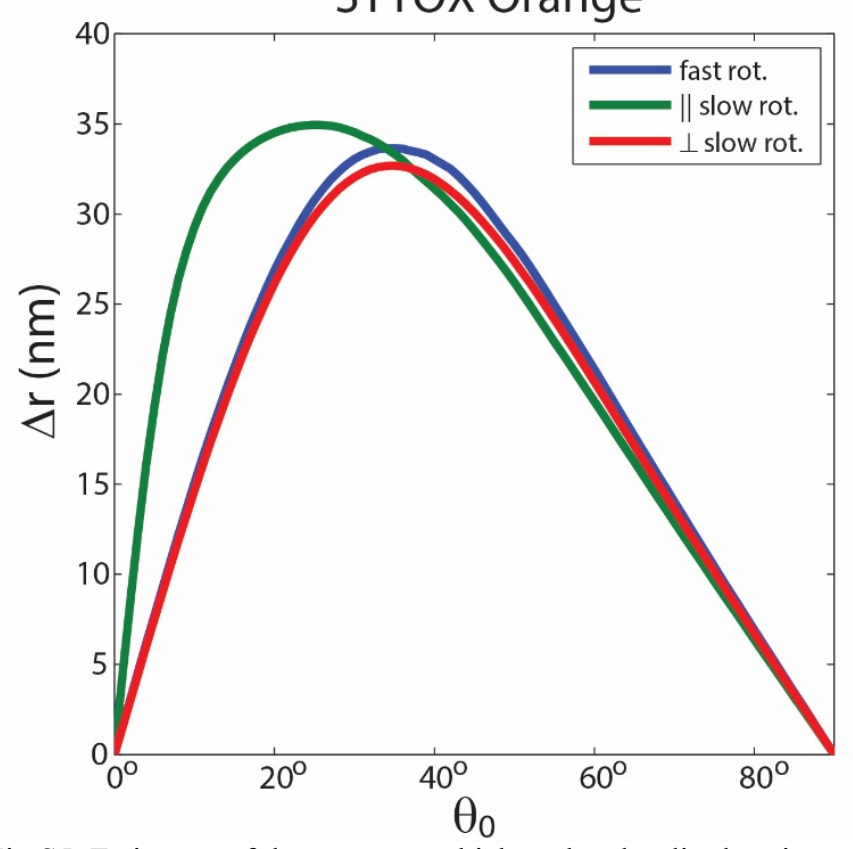

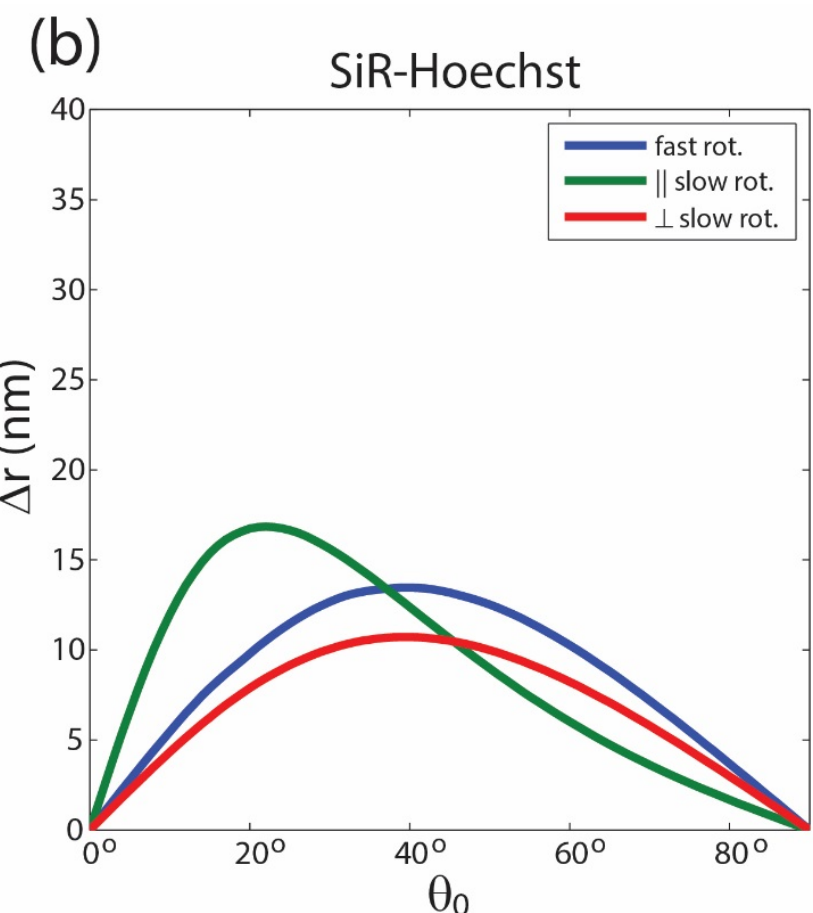

Fig S5. Estimates of the extent to which molecular dipole orientation contributes to localization errors as described in section 6 . Calculations were performed for fast $\left(\tau_{f} \gg \tau_{r}\right)$ and slow $\left(\tau_{f}<<\tau_{r}\right)$ rotational diffusion. When assuming slow rotational diffusion, we investigate the cases of excitation parallel $(\|)$ and perpendicular $(\perp)$ to the molecule's mean azimuthal orientation $\phi_{0}$.

(a) Estimating an average microscope defocus of $100 \mathrm{~nm}$, and a cone-angle $\alpha=22^{\circ}$, we plot the localization errors incurred when using the dye SYTOX Orange. A maximum error of $35 \mathrm{~nm}$ is realized. (b) For the dye SiR-Hoechst, a significantly larger cone angle of $\alpha=58.5^{\circ}$ yields a maximum localization error of $16 \mathrm{~nm}$.

\section{References}

1. X. Michalet, R. Ekong, F. Fougerousse, S. Rousseaux, C. Schurra, N. Hornigold, M. Slegtenhorst, J. Wolfe, S. Povey, J. S. Beckmann, and A. Bensimon, "Dynamic Molecular Combing:Stretching the Whole Human Genome for High-Resolution Studies," Science 277, 1518-1522 (1997).

2. C. Flors, C. Ravarani, and D. Dryden, "Super-resolution imaging of DNA labelled with intercalating dyes," ChemPhysChem 10, 2201-2204 (2009).

3. I. Schoen, J. Ries, E. Klotzsch, H. Ewers, and V. Vogel, "Binding-Activated Localization Microscopy of DNA Structures," Nano Lett. 11, 4008-4011 (2011).

4. H. Miller, Z. Zhou, A. Wollman, and M. C. Leake, "Superresolution imaging of single DNA molecules using stochastic photoblinking of minor groove and intercalating dyes," Methods 88, 81-88 (2015).

5. H. Sosa, E. J. G. Peterman, W. E. Moerner, and L. S. B. Goldstein, "ADP-Induced Rocking of the Kinesin Motor Domain Revealed by Single-Molecule Fluorescence Polarization Microscopy," Nat. Struct. Biol. 8, 540-544 (2001).

6. E. J. G. Peterman, H. Sosa, L. S. B. Goldstein, and W. E. Moerner, "Polarized Fluorescence Microscopy of Individual and Many Kinesin Motors Bound to Axonemal Microtubules," Biophys. J. 81, 2851-2863 (2001).

7. R. E. Thompson, D. R. Larson, and W. W. Webb, "Precise Nanometer Localization Analysis for Individual Fluorescent Probes," Biophys. J. 82, 2775-2783 (2002).

8. M. A. Thompson, "The development of techniques for three-dimensional super-resolution fluorescence microscopy and their application to biological systems," Ph. D. Thesis, Stanford University (2011).

9. E. Hoogendoorn, K. C. Crosby, D. Leyton-Puig, R. M. Breedijk, K. Jalink, T. W. Gadella, and M. Postma, "The fidelity of stochastic single-molecule superresolution reconstructions critically depends upon robust background estimation," Scientific reports 4, (2014).

10. R. Henriques, M. Lelek, E. F. Fornasiero, F. Valtorta, C. Zimmer, and M. M. Mhlanga, "QuickPALM: 3D real-time photoactivation nanoscopy image processing in ImageJ," Nat. Methods 7, 339-340 (2010).

11. Y. Wang, J. Schnitzbauer, Z. Hu, X. Li, Y. Cheng, Z. Huang, and B. Huang, "Localization events-based sample drift correction for localization microscopy with redundant cross-correlation algorithm," Opt. Express 22, 15982-15991 (2014).

12. T. Ha, J. Glass, T. Enderle, D. S. Chemla, and S. Weiss, "Hindered Rotational Diffusion and Rotational Jumps of Single Molecules," Phys. Rev. Lett. 80, 20932096 (1998).

13. M. D. Lew, M. P. Backlund, and W. E. Moerner, "Rotational Mobility of Single Molecules Affects Localization Accuracy in Super-Resolution Fluorescence Microscopy," Nano Lett. 13, 3967-3972 (2013).

14. S. Stallinga, "Effect of rotational diffusion in an orientational potential well on the point spread function of electric dipole emitters," JOSA A 32, 213-223 (2015).

15. A. S. Backer and W. E. Moerner, "Determining the rotational mobility of a single molecule from a single image: a numerical study," Opt. Express 23, 42554276 (2015) 
16. J. N. Forkey, M. E. Quinlan, M. A. Shaw, J. E. Corrie, and Y. E. Goldman, "Three-dimensional structural dynamics of myosin V by single-molecule fluorescence polarization," Nature 422, 399-404 (2003).

17. J. N. Forkey, M. E. Quinlan, and Y. E. Goldman, "Measurement of Single Macromolecule Orientation by Total Internal Reflection Fluorescence Polarization Microscopy," Biophys. J. 89, 1261-1271 (2005).

18. M. E. Quinlan, J. N. Forkey, and Y. E. Goldman, "Orientation of the Myosin Light Chain Region by Single Molecule Total Internal Reflection Fluorescence Polarization Microscopy," Biophys. J. 89, 1132-1142 (2005).

19. S. A. Rosenberg, M. E. Quinlan, J. N. Forkey, and Y. E. Goldman, "Rotational motions of macro-molecules by single-molecule fluorescence microscopy," Acc. Chem. Res. 38, 583-593 (2005).

20. J. Enderlein, E. Toprak, and P. R. Selvin, "Polarization effect on position accuracy of fluorophore localization," Opt. Express 14, $8111-8120$ (2006).

21. S. Stallinga and B. Rieger, "Accuracy of the Gaussian point spread function model in 2D localization microscopy," Opt. Express 18, 24461-24476 (2010).

22. J. Engelhardt, J. Keller, P. Hoyer, M. Reuss, T. Staudt, and S. W. Hell, "Molecular orientation affects localization accuracy in superresolution far-field fluorescence microscopy," Nano Lett. 11, 209-213 (2011).

23. A. S. Backer and W. E. Moerner, "Extending Single-Molecule Microscopy Using Optical Fourier Processing," J. Phys. Chem. B 118, 8313-8329 (2014).

24. S. Stallinga and B. Rieger, "Position and orientation estimation of fixed dipole emitters using an effective Hermite point spread function model," Opt. Express 20, 5896-5921 (2012).

25. M. P. Backlund, M. D. Lew, A. S. Backer, S. J. Sahl, G. Grover, A. Agrawal, R. Piestun, and W. E. Moerner, "Simultaneous, accurate measurement of the 3D position and orientation of single molecules," Proc. Natl. Acad. Sci. U. S. A. 109, 19087-19092 (2012).

26. M. D. Lew and W. E. Moerner, "Azimuthal Polarization Filtering for Accurate, Precise, and Robust Single-Molecule Localization Microscopy," Nano Lett. 14, 6407-6413 (2014) 